\title{
THE EXISTENCE OF GAUGE FORMS ON AFFINE VARIETIES
}

\author{
RICHARD L. FABER
}

1. Introduction. One way of defining the Tamagawa number of a linear algebraic group $G$ is to start with a gauge form [1] on $G$, i.e., an invariant highest degree algebraic differential form which is everywhere holomorphic and nonzero. In this paper we consider the existence of an everywhere holomorphic and nonzero form of highest degree on an arbitrary nonsingular affine variety $V$ defined over an arbitrary field $k$. It is proved that a variety which is a complete intersection possesses a gauge form. Some comments are made on the general case.

2. Preliminaries. Let $V \subset \Omega^{n}$ be a nonsingular $r$-dimensional variety defined over a field $k$ contained in a universal domain $\Omega$. If $x=\left(x_{1}, x_{2}\right.$, $\left.\cdots, x_{n}\right)$ is a generic point for $V$ over $k$, we identify $k(x)$ with the function field, $k(V)$, of $V$, and let $D$ be the $k(x)$-space of $k$-derivations of $k(x)$. The elements of $\mathcal{F}=\operatorname{Hom}_{k(x)}(D, k(x))$ are called algebraic differential 1-forms over $k$ on $V$; the elements of $\Lambda^{r} \mathfrak{F}$ are the algebraic differential $r$-forms over $k$ on $V$, or briefly, the $r$-forms over $k$. We define the map $d: k(x) \rightarrow \mathcal{F}$ by $d f(x)(D)=D f(x)$ for $f(x) \in k(x), D \in D$.

Let $x^{\prime}$ be a point of $V$. A separating transcendence base $t_{1}, t_{2}$, $\cdots, t_{r}$ for $k(x)$ over $k$ is called a local coordinate system at $x^{\prime}$ if, for each $i$,

(1) $t_{i} \in \mathcal{O}_{x^{\prime}}$, the local ring at $x^{\prime}$, and

(2) the derivation $\partial / \partial t_{i}$ maps $\mathcal{O}_{x^{\prime}}$ into itself. (2) is equivalent to the statement that $\partial x_{j} / \partial t_{i} \in \mathcal{O}_{x^{\prime}}$, for all $j$. Given such a local system, any $r$-form $\omega$ has a "local expression" at $x^{\prime}$ of the form

$$
\omega=\phi(x) d t_{1} \wedge d t_{2} \wedge \cdots \wedge d t_{r},
$$

for some $\phi(x) \in k(x)$. $\omega$ is called a gauge form (over $k$ ) on $V$ if at every point $x^{\prime}$ of $V$ it has such a local expression in which $\phi(x) \in \Theta_{x^{\prime}}$ and $\phi\left(x^{\prime}\right) \neq 0$, i.e., $\phi(x) \in \mathcal{O}_{x^{\prime}}^{*}$, the group of units in $\Theta_{x^{\prime}}$.

3. Complete intersections. Let $V \subset \Omega^{r+N}$ be a nonsingular $r$-dimensional affine variety defined over $k$ by equations $F_{1}, F_{2}, \cdots, F_{N}$ in $k[X]=k\left[X_{1}, \cdots, X_{r+N}\right]$ and let $x=\left(x_{1}, x_{2}, \cdots, x_{r+N}\right)$ be generic over $k$. If $\left\{i_{1}, i_{2}, \cdots, i_{r}\right\}$ is a subset of $\{1,2, \cdots, r+N\}$, we shall denote its complement by $\left\{i_{1}^{\prime}, i_{2}^{\prime}, \cdots, i_{N}^{\prime}\right\}$.

Presented to the Society, October 28, 1967 ; received by the editors June 12, 1967. 
Now let $x^{\prime}$ be any point of $V$, and suppose

$$
\partial\left(F_{1}, \cdots, F_{N}\right) / \partial\left(X_{i^{\prime} 1}, \cdots, X_{i^{\prime} N}\right)_{x^{\prime}} \neq 0 .
$$

Then the same determinant evaluated at the generic point does not vanish, and so $x_{i_{1}}, \cdots, x_{i_{r}}$ is a separating transcendence base for $k(x)$ over $k$. To show it is a system of local coordinates at $x^{\prime}$, it suffices to prove that $\partial x_{i^{\prime} \mu} / \partial x_{i_{\lambda}} \in \mathcal{O}_{x^{\prime}}$ for $1 \leqq \mu \leqq N, 1 \leqq \lambda \leqq r$. But since $d x_{i_{1}}$, $\cdots, d x_{i_{r}}$ are a basis for the space of 1 -forms,

$$
d x_{i^{\prime}{ }_{\mu}}=\sum_{\lambda=1}^{r}\left(\partial x_{i^{\prime}{ }^{\prime}} / \partial x_{\dot{i}_{\lambda}}\right) d x_{\dot{i}^{\prime}}, \quad 1 \leqq \mu \leqq N .
$$

Moreover, for each $m=1,2, \cdots, N$, the equation $0=d F_{m}(x)$ implies

$$
-\sum_{\mu=1}^{N}\left(\partial F_{m} / \partial X_{i^{\prime} \mu_{\mu}}\right)_{x} d x_{i^{\prime}{ }_{\mu}}=\sum_{\lambda=1}^{r}\left(\partial F_{m} / \partial X_{i_{\lambda}}\right)_{x} d x_{i_{\lambda}} .
$$

Since det $\left(\left(\partial F_{m} / \partial X_{i^{\prime} \mu}\right)\right)_{x} \in \mathcal{O}_{x}^{*}$, the result follows from (1) and (2).

Now denote by $\sigma(i)$ the sign of the permutation sending $(1,2, \cdots$, $r+N)$ into $\left(i_{1}, i_{2}, \cdots, i_{r}, i_{1}^{\prime}, \cdots, i_{N}^{\prime}\right)$, and let

$$
\omega^{\prime}=\sigma(i) \frac{d x_{i_{1}} \wedge d x_{i_{2}} \wedge \cdots \wedge d x_{i_{r}}}{\partial\left(F_{1}, \cdots, F_{N}\right) / \partial\left(X_{i^{\prime}{ }_{1}}, \cdots, X_{i^{\prime}{ }_{N}}\right)_{x}} .
$$

This is an $r$-form over $k$ on $V$, holomorphic and nonzero at $x^{\prime}$. We shall show that $\omega^{\prime}$ is independent of the choice of $x^{\prime}$ and local coordinates at $x^{\prime}$. It will then follow that $\omega^{\prime}$ is a gauge form.

Let $x^{\prime \prime}$ be any point of $V$ (possibly equal to $x^{\prime}$ ), and suppose that $\partial\left(F_{1}, \cdots, F_{N}\right) / \partial\left(X_{j^{\prime}}, \cdots, X_{j^{\prime} N}\right)_{x^{\prime \prime}} \neq 0$, so that $x_{j_{1}}, \cdots, x_{j_{r}}$ is a system of local coordinates at $x^{\prime \prime}$. Set

$$
\omega^{\prime \prime}=\sigma(j) \frac{d x_{j_{1}} \wedge d x_{j_{2}} \wedge \cdots \wedge d x_{j_{r}}}{\partial\left(F_{1}, \cdots, F_{N}\right) / \partial\left(X_{j^{\prime} 1}, \cdots, X_{j^{\prime} N}\right)_{x}} .
$$

We claim that $\omega^{\prime}=\omega^{\prime \prime}$. This is equivalent to the assertion

$$
\begin{aligned}
\partial\left(x_{j_{1}}, \cdots, x_{j_{r}}\right) / \partial\left(x_{i_{1}}, \cdots, x_{i_{r}}\right) \\
=\sigma(i) \sigma(j) \frac{\partial\left(F_{1}, \cdots, F_{N}\right) / \partial\left(X_{j^{\prime}}, \cdots, X_{j^{\prime} N_{N}}\right)_{x}}{\partial\left(F_{1}, \cdots, F_{N}\right) / \partial\left(X_{i^{\prime} 1}, \cdots, X_{i^{\prime}{ }_{N}}\right)_{x}} .
\end{aligned}
$$

But the latter is an elementary result of the calculus.

4. The general case. Let $\left\{F_{\alpha}\right\}_{a \in A}$ be any collection of polynomials in $k[X]$ generating the ideal of $V \subset \Omega^{n}$ in $\Omega[X]$. We denote by $B$ the set of all $N$-tuples ( $N$ is the codimension) $\beta=\left(\beta_{1}, \beta_{2}, \cdots, \beta_{N}\right)$ of 
elements of $A$ such that the matrix with $i$ th row $\partial F_{\beta_{i}} / \partial X_{1}, \cdots$, $\partial F_{\beta_{i}} / \partial X_{n}$, for $i=1,2, \cdots, N$, has rank $N$ at $x$. For each $\beta \in B$, let $U_{\beta}=\left\{x^{\prime} \in V \mid\right.$ the rank of the same matrix evaluated at $x^{\prime}$ is also $\left.N\right\}$. Then the $\left\{U_{\beta}\right\}_{\beta \in B}$ are a $k$-open cover of $V$.

For $\beta, \gamma \in B$, set $c_{\beta}^{\gamma}=\operatorname{det} C_{\beta}^{\gamma}$, where the matrix with $i$ th row $\partial F_{\gamma_{i}} / \partial X_{1}, \cdots, \partial F_{\gamma_{i}} / \partial X_{n}$ evaluated at $x$ is $C_{\beta}^{\gamma}$ times the matrix with $i$ th row $\partial F_{\beta_{i}} / \partial X_{1}, \cdots, \partial F_{\beta_{i}} / \partial X_{n}$ evaluated at $x$. Then $C_{\beta}^{\gamma}$ is an $N \times N$ matrix over $k(x)$ and $c_{\beta}^{\gamma} \in \mathcal{O}^{*}\left(U_{\beta} \cap U_{\gamma}\right)$.

It is easily seen that $\left\{c_{\beta}^{\gamma}\right\}_{\beta, \gamma \in B}$ is a 1-cocycle for the cohomology of the cover $\left\{U_{\beta}\right\}_{\beta \in B}$ of $V$ with coefficients in the sheaf of local units. Moreover, it is not hard to prove that the element represented in the Cech cohomology $H^{1}\left(V, \mathcal{O}_{V}^{*}\right)$ is zero if and only if $V$ possesses a gauge form over $k$.

We shall now give an example, due to D. S. Rim, of a nonsingular affine variety which possesses no gauge form. Suppose that $R$ is the coordinate ring of an $r$-dimensional variety $V$ defined over $k$, and that $S$ is the coordinate ring of an $s$-dimensional variety $W$ defined over $k$. Assume that $S$ is an augmented $R$-algebra.

Let $\mathcal{F}_{R}$ be the $R$-module of all 1 -forms over $k$ on $V$ which are everywhere holomorphic; $\mathcal{F}_{S}$, the $S$-module of all 1 -forms over $k$ on $W$ which are everywhere holomorphic. Finally, let $\mathfrak{F}_{S / R}$ denote the $S$-module consisting of all elements of $\operatorname{Hom}_{k(W)}\left(\operatorname{Der}_{R} k(W), k(W)\right)$ which are holomorphic everywhere on $W$. We have natural $k$-derivations $d_{R}: R \rightarrow \mathcal{F}_{R}, d_{S}: S \rightarrow \mathcal{F}_{S}$, and a natural $S$-derivation $d_{S / R}: S \rightarrow \mathcal{F}_{S / R}$, all satisfying $(d f) D=D f$, for $f$ in $R$ or $S$ and $D$ in $\mathcal{F}_{R}, \mathcal{F}_{S}$, or $\mathcal{F}_{S / R}$, as the case may be. These derivations yield canonical isomorphisms

$$
\begin{aligned}
& \operatorname{Hom}_{R}\left(\mathfrak{F}_{R}, M\right) \approx \operatorname{Der}_{k}(R, M), \quad \text { for any } R \text {-module } M, \\
& \operatorname{Hom}_{S}\left(\mathfrak{F}_{S}, M\right) \approx \operatorname{Der}_{k}(S, M), \quad \text { for any } S \text {-module } M, \\
& \operatorname{Hom}_{S}\left(\mathfrak{F}_{S / R}, M\right) \approx \operatorname{Der}_{R}(S, M), \quad \text { for any } S \text {-module } M .
\end{aligned}
$$

Now, since $S$ is an augmented $R$-algebra,

$$
0 \rightarrow \operatorname{Der}_{R}(S, M) \rightarrow \operatorname{Der}_{k}(S, M) \rightarrow \operatorname{Der}_{k}(R, M) \rightarrow 0
$$

is exact for any $S$-module $M$. Moreover,

$$
0 \rightarrow S \otimes_{R} \mathfrak{F}_{R} \rightarrow \mathfrak{F}_{S} \rightarrow \mathfrak{F}_{S / R} \rightarrow 0
$$

is a split exact sequence of $S$-modules. Exactness is seen by applying $\operatorname{Hom}_{S}(-, M$ ) for any $S$-module $M$ (by (5) and (6), we always obtain an exact sequence), and splitness is seen by taking $M=S \otimes_{R} \mathcal{F}_{R}$.

Let $\left(\Lambda^{\circ} \mathcal{F}\right)_{S}$ be the $S$-module of everywhere holomorphic $s$-forms over $k$ on $W$. Then the natural map $\Lambda^{*}\left(F_{S}\right) \rightarrow\left(\Lambda^{*} \mathcal{F}\right)_{S}$ is an isomor- 
phism, since it is such locally. Clearly, if there exists a gauge form $\omega$ on $W$, then $\left(\Lambda^{*} \mathcal{F}\right)_{S}$ is a free $S$-module, $S \omega$; and, conversely, if $\left(\Lambda^{*} \mathscr{F}\right)_{S}$ is nontrivial free, then $W$ possesses a gauge form (any generator of $\left.\left(\Lambda^{8} \mathcal{F}\right)_{S}\right)$. We now construct a variety $W$ for which $\Lambda^{*}\left(\mathcal{F}_{S}\right)$ is not free.

Take $k=\Omega=$ the field of complex numbers, and let $R$ be the quotient ring of the polynomial ring $\Omega[X, Y, Z]$ by the ideal generated by $X^{2}+Y^{2}+Z^{2}-1$. Since $R$ is not a unique factorization domain $((X+i Y)(X-i Y)=-(Z-1)(Z+1))$, there exists in $R$ an ideal $\mathfrak{A}$ which is locally principal but not principal, e.g., $\mathfrak{A}=R(X+i Y)(Z-1)$.

Let $S=\delta(\mathfrak{A})$, the symmetric algebra on $\mathfrak{A}$ (it is locally a polynomial ring in one variable). Then $V$ is the 2 -sphere, and $W$ is a nonsingular affine variety which is locally $V \times$ a straight line.

Now, from (7) and the fact that $\operatorname{dim} W=3, \operatorname{dim} V=2$, we obtain

$$
\Lambda^{3}\left(F_{S}\right) \approx \Lambda^{2}\left(S \otimes_{R} F_{R}\right) \otimes_{S} \mathfrak{F}_{S / R} \approx S \otimes_{S} \mathfrak{F}_{S / R} \approx \mathfrak{F}_{S / R} .
$$

It will therefore suffice to prove that $\mathfrak{F}_{S / R}$ is not $S$-free.

However, we have the following sequence of natural transformations: for any $S$-module $M, \operatorname{Hom}_{S}\left(\mathcal{F}_{S / R}, M\right) \approx \operatorname{Der}_{R}(S, M) \approx$ $\operatorname{Hom}_{R}(\mathfrak{A}, \quad M) \approx \operatorname{Hom}_{S}\left(S \otimes_{R} \mathfrak{A}, M\right)$. Hence, $\mathfrak{F}_{S / R} \approx S \otimes_{R} \mathfrak{A}$, canonically. But $S \otimes_{R} \mathfrak{A}$ is not $S$-free; for, if it were (viewing $R$ as an $S$ module), $R \otimes_{S}\left(S \otimes_{R} \mathfrak{A}\right) \approx R \otimes_{R} \mathfrak{A} \approx \mathfrak{A}$ would be $R$-free, a contradiction. Accordingly, $W$ possesses no gauge form.

\section{REFERENCE}

1. A. Weil, Adeles and algebraic groups, Notes, Institute for Advanced Study, Princeton, N. J., 1961.

University of California at San Diego 